\title{
BMJ Open Impact of biological agents and tofacitinib for moderate-to-severe ulcerative colitis on health-related quality of life: a protocol for a network meta-analysis
}

Xueni Ma (D) , ${ }^{1}$ Huimei Xu, ${ }^{1}$ Dekui Zhang ${ }^{2}$

To cite: Ma X, Xu H, Zhang D. Impact of biological agents and tofacitinib for moderateto-severe ulcerative colitis on health-related quality of life: a protocol for a network meta-analysis. BMJ Open 2021;11:e055182. doi:10.1136/ bmjopen-2021-055182

- Prepublication history and additional supplemental material for this paper are available online. To view these files, please visit the journal online (http://dx.doi.org/10.1136/ bmjopen-2021-055182).

Received 08 July 2021 Accepted 03 December 202

A) Check for updates

(c) Author(s) (or their employer(s)) 2021. Re-use permitted under CC BY-NC. No commercial re-use. See rights and permissions. Published by BMJ.

${ }^{1}$ The Second Clinical Medical College of Lanzhou University, Lanzhou University, Lanzhou,

Gansu, China

${ }^{2}$ Department of

Gastroenterology, Lanzhou University Second Hospital, Lanzhou, Gansu, China

Correspondence to Professor Dekui Zhang; zhangdk8616@126.com

\section{ABSTRACT}

Introduction In the past few years, several options have been proposed as alternative and more effective therapeutic drugs for moderate-to-severe ulcerative colitis (UC), such as biological agents and tofacitinib. Most of the clinical studies related to UC aimed to evaluate the efficacy of the drugs on clinical outcomes such as disease activity and side effects. This review aims to compare the impact of infliximab, adalimumab, golimumab, ustekinumab, vedolizumab and tofacitinib for moderate-to-severe UC on health-related quality of life (HRQDL), thereby guiding clinical treatment and scientific research of this disease. Methods and analysis We will search PubMed, Embase and Cochrane Library from inception until July 2021 for all randomised controlled trials (RCTs) reported in English as double-blind comparing infliximab, adalimumab, golimumab, ustekinumab, vedolizumab or tofacitinib as induction or maintenance therapies with another or with placebo in moderate-to-severe UC on HRQoL. The primary outcome of this study is changes in the mean difference in $\mathrm{HRQOL}$ scores. Data of each pairwise comparison will be synthesised to obtain summary standardised mean differences for continuous outcomes and ORs for dichotomous outcomes. Then, a network meta-analysis (NMA) will be performed, and a commoneffects Mantel-Haenszel NMA will be conducted for dichotomous outcomes, while a random-effects NMA will be used for all other outcomes. Finally, we will follow the Grading of Recommendations, Assessment, Development and Evaluations approach to assess the confidence in estimates derived from NMA of the main outcomes. Ethics and dissemination Only published secondary data will be used in this study, and therefore ethics approval is not required. The findings will be published in a peerreviewed medical journal.

PROSPERO registration number CRD42021225048.

\section{INTRODUCTION}

Ulcerative colitis (UC) is a chronic and lifelong condition characterised by inflammation and ulceration of the rectal and colonic mucosa. Patients with UC typically manifest recurring and episodic clinical symptoms,
Strengths and limitations of this study

- This network meta-analysis will provide a comprehensive summary of the direct and indirect evidence on the impact of biological agents and tofacitinib for moderate-to-severe ulcerative colitis on healthrelated quality of life (HRQoL).

- We will follow the Grading of Recommendations, Assessment, Development and Evaluations approach to assess the confidence in estimates derived from the network meta-analysis of the main outcomes.

- We will assess the HRQoL of individuals with moderate-to-severe ulcerative colitis who received biological agents or tofacitinib to inform clinicians and the development of guidelines for the management of those patients.

- It is expected that this systematic review will encourage further research to be conducted for which systematic review evidence of high quality is currently insufficient or lacking.

- Possible limitations of this review include the diverse outcome measurements, the possible role of confounding factors in biasing finding estimates and the limited data available for some interventions.

including rectal bleeding, bloody diarrhoea, urgency, tenesmus and abdominal pain. ${ }^{1}$ In recent decades, many studies have reported that the incidence and prevalence of UC increased in Asia, making it a kind of global disease. ${ }^{2}{ }^{3}$ The physical health and mental health of patients with $\mathrm{UC}$ are poorer than those of healthy subjects, posing a great direct and indirect burden on the patient and society. ${ }^{4}$ Therefore, given that the physical and emotional impact of UC causes a huge burden on patients' daily functioning and well-being, their health-related quality of life (HRQoL) is severely compromised. ${ }^{5}$ Recently, the US Food and Drug Administration (FDA) and the European Medicines 
Agency (EMA) have suggested that HRQoL can be an important measure in drug development clinical trials. ${ }^{67}$

Conventional treatments of UC, including 5-aminosalicylate, glucocorticoids and immunomodulators, have high rates of relapse. ${ }^{8}$ In the past few years, several options have been proposed as alternative and more effective therapeutic drugs for moderate-to-severe UC, such as anti-tumour necrosis factor $\alpha$ (anti-TNF- $\alpha$ ) and anti-integrin antibodies. ${ }^{9-13}$ The efficacy and safety of infliximab, adalimumab, golimumab, ustekinumab, vedolizumab and tofacitinib in patients with moderate-to-severe active UC have been evaluated in recent studies. ${ }^{1415}$ In the clinical trials of inflammatory bowel disease, outcomes reported by patients are mainly focused on the efficacy of drugs such as disease activity and side effects. However, as a useful index for guiding future clinical decision-making and healthcare policy, the HRQoL of patients with UC is less presented. The generic HRQoL measures contain the Short-Form 36 questionnaire (SF-36) and European Quality of Life-5 Dimensions (EQ-5D), while the Inflammatory Bowel Disease Questionnaire (IBDQ) is a diseasespecific questionnaire. ${ }^{16}$ A network meta-analysis (NMA) suggested that compared with placebo, induction treatment with infliximab, golimumab, adalimumab, vedolizumab and tofacitinib improves patients' quality of life. ${ }^{17}$ However, ustekinumab, a human IgG1 monoclonal antibody, has been approved for treating many diseases, such as psoriasis, psoriatic arthritis, ${ }^{18}$ Crohn's disease ${ }^{19}$ and UC recently. ${ }^{20}$ Moreover, the efficacy and safety of ustekinumab in patients with moderate-to-severe UC have been evaluated in a phase III, randomised, double-blinded, placebo-controlled study. ${ }^{20}$ Although a head-to-head clinical trial has been conducted to compare vedolizumab versus adalimumab in patients with moderate-to-severe UC, the lack of head-to-head trials renders that the existing evidence cannot provide a basis for a more effective comprehensive decision-making. ${ }^{21}$ Given the availability of a new drug, ustekinumab, for the treatment of $\mathrm{UC}$ and the publication of a head-to-head pilot study, a new NMA is warranted to further provide a comprehensive basis for biological agents and tofacitinib in the treatment of UC.

Therefore, we will conduct a systematic review and NMA to compare the impact of infliximab, adalimumab, golimumab, ustekinumab, vedolizumab and tofacitinib for moderate-to-severe UC on HRQoL, thereby guiding for clinical treatment and scientific research of this disease.

\section{METHODS AND ANALYSIS}

The protocol of this systematic review was registered in the International Prospective Register of Systematic Reviews (PROSPERO). We will adhere to the Preferred Reporting Items for Systematic Review and Meta-Analyses (PRISMA) and the guideline for systematic review and meta-analysis protocols (PRISMA-P), as well as the extension statement for NMA (PRISMA-NMA). ${ }^{22} 23$

\section{Data source and search strategy}

We will search the PubMed, Embase and Cochrane Library from inception until July 2021 for all randomised controlled trials (RCTs) reported as double-blind to compare infliximab, adalimumab, golimumab, ustekinumab, vedolizumab and tofacitinib as induction or maintenance therapies with another or with placebo in moderate-to-severe UC on HRQoL. ClinicalTrials.gov and the websites of the EMA and the FDA for ongoing or completed clinical trials will also be searched. Our search will be limited to papers published in international scientific journals and English. The databases will be searched using search terms related to ulcerative colitis, proctitis, IBD, anti-TNF, infliximab, adalimumab, golimumab, tofacitinib, ustekinumab, anti-alpha4, Janus Kinases and RCTs. The search strategy is listed in online supplemental file 1. Furthermore, we will search for additional RCTs in the reference lists of included studies.

\section{Eligibility criteria \\ Population}

Adults (aged $>18$ years) with moderate-to-severe UC (Mayo Clinic score of 6-12, with an endoscopic subscore of 2 or 3) who were either treatment-naive (first-line) or previously exposed to TNF- $\alpha$ antagonists (second-line) will be included in this study.

\section{Interventions and comparisons}

This review will include interventions including infliximab, adalimumab, golimumab, vedolizumab, ustekinumab or tofacitinib, and the treatment courses of greater than 2 weeks. Comparisons may include another intervention as mentioned above or placebo.

\section{Outcomes}

The primary outcome of this study is changes in the mean difference in HRQoL scores. We will measure the primary outcome at the end of induction therapy (6-8 weeks) and the end of maintenance therapy (approximately 1 year). The HRQoL measures considered in this study are SF-36, EQ-5D and IBDQ. Secondary outcomes are the proportion of patients who achieved clinically significant improvement in the HRQoL (as defined by the investigators of each study) and the proportion of patients with an IBDQ total score $\geq 170$ points.

\section{Study designs}

We will include RCTs that provide at least one comparison between two interventions of interest. In addition, we will not limit study inclusion by publication status and period in which the study has been conducted.

\section{Study selection}

Literature search records will be imported into EndNote V.X9 (Thomson Reuters, New York, NY, USA) literature management software. Two reviewers will independently read the titles and abstracts of retrieved studies according to the inclusion criteria to identify potentially eligible RCTs for inclusion. The same two reviewers will obtain 
the full-text reports of potentially eligible studies and independently assess those studies for inclusion or exclusion. Disagreements will be resolved by a third reviewer.

\section{Data extraction}

Data will be extracted using a prespecified Microsoft Excel collection form. Two reviewers will independently extract detailed information from those included articles. Discrepancies will be resolved through team discussion or consulting a third investigator. The following information will be extracted from each study: study characteristics, participants' baseline characteristics and HRQoL outcomes. Data from different reports for the same study will be collated.

\section{Risk of bias assessment among included studies}

To evaluate the risk of bias, we will use the Cochrane Collaboration revised tool to assess risk of bias in randomised trials (RoB V.2.0). ${ }^{24}{ }^{25}$ Two independent reviewers will perform the evaluation using the provided Microsoft Excel form. If disagreements occur, the final result will be made by consensus with the involvement of another member of the review group. Studies reporting at least one primary outcome will be evaluated in the following domains: sequence for random allocation, allocation concealment, blinding of study personnel and participants, blinding of outcome assessment, incomplete outcome data, selective reporting and other biases such as sponsorship bias. Studies that meet all the criteria were rated as 'low' risk, while studies with insufficient procedures in at least one domain were rated as 'high' risk. In all other cases, studies were rated to be at 'unclear' risk of bias. ${ }^{25}$

\section{Statistical synthesis of study data \\ Pairwise meta-analyses}

The characteristics of the included RCTs will be summarised. We will synthesise data of each pairwise comparison to obtain summary standardised mean differences for continuous outcomes and ORs for dichotomous outcomes, both with $95 \%$ CIs. Statistical heterogeneity across trials will be evaluated by $\mathrm{I}^{2}$ statistics and Cochran's $Q$ test. $\mathrm{I}^{2}$ statistic with values $>50 \%$ or $\mathrm{p}<0.10$ for Cochran's $\mathrm{Q}$ test suggested significant heterogeneity. ${ }^{26}$ Data from all included trials were pooled to assess the overall effect on HRQoL of any active treatment compared with placebo.

\section{Network meta-analysis}

R V.4.0.4 and STATA V.15.1 (Stata Corp, College Station, TX, USA) were used for NMA. For dichotomous outcomes, we will perform a common-effects MantelHaenszel NMA. ${ }^{27}$ For all other outcomes, we will employ a random-effects NMA given a single heterogeneity parameter for different comparisons in each network. Each node represents a treatment option, and the size of nodes is relevant to the sample size of each treatment in network diagrams, and the line between two certain nodes indicates that a direct comparison between the two treatment regimens exists; also the width of lines is related to the number of studies. ${ }^{14}$ For each outcome, we will use the rankogram plots and the surface under the cumulative ranking curves (SUCRA) to figure out the hierarchy of different therapies. The probabilities of therapies will be presented by the rankogram plots supposing any of the possible ranks. Furthermore, SUCRA curves will be shown as percentages, $100 \%$ for the best treatment and $0 \%$ for the worst treatment. ${ }^{28}$

\section{Sensitivity analyses}

To understand how the following factors influence the effect estimates, we will perform sensitivity analysis by excluding studies with a high risk of bias in each assessed item. In addition, we will conduct a sensitivity analysis by excluding those studies where very high/low dosages were used for off-label medications.

\section{Assessment of inconsistency}

The consistency assumption will be assessed for each comparison where there is direct and indirect evidence and the two will be compared. Furthermore, 'design-bytreatment interaction' models will be used to examine the consistency across the whole network, which carry out an overall significance test for inconsistency.

\section{Assessment of publication bias}

For the primary outcome, we will use Egger's test and funnel plots to assess the publication bias of the included studies. We will try to explain funnel plot asymmetry if the funnel plots are found to be asymmetrical. ${ }^{29}$

\section{Grading the quality of evidence}

We will follow the Grading of Recommendations, Assessment, Development and Evaluations approach to assess the confidence in estimates derived from the NMA of the main outcomes. In this method, direct evidence from RCTs will be rated as high confidence and can be downgraded according to the following five domains-risk of bias, indirectness, imprecision, inconsistency and publication bias-to levels of moderate, low and very low confidence. $^{3031}$

\section{Patient and public involvement}

There was no patient or public involvement in the design of this study, or conduct, or reporting or dissemination plans of this manuscript.

\section{Ethics and dissemination}

This work relies on published data and therefore does not require an ethical approval. The findings will be published in a scientific peer-reviewed journal.

Contributors XM and DZ conceived the idea for this study; DZ designed the metaanalysis; XM and $\mathrm{HX}$ provided statistical advice and input; XM drafted the protocol. $\mathrm{DZ}$ and $\mathrm{HX}$ reviewed the protocol and provided critical feedback. All authors approved the manuscript in its final form.

Funding This work is supported by the National Natural Science Foundation of China (81770525), the Health Research Programme Project of Gansu Province (GSWSKY2017-01) and the Talent Innovation and Entrepreneurship Project of Lanzhou (2018-RC-76). 
Competing interests None declared.

Patient consent for publication Not applicable.

Provenance and peer review Not commissioned; externally peer reviewed.

Supplemental material This content has been supplied by the author(s). It has not been vetted by BMJ Publishing Group Limited (BMJ) and may not have been peer-reviewed. Any opinions or recommendations discussed are solely those of the author(s) and are not endorsed by BMJ. BMJ disclaims all liability and responsibility arising from any reliance placed on the content. Where the content includes any translated material, BMJ does not warrant the accuracy and reliability of the translations (including but not limited to local regulations, clinical guidelines, terminology, drug names and drug dosages), and is not responsible for any error and/or omissions arising from translation and adaptation or otherwise.

Open access This is an open access article distributed in accordance with the Creative Commons Attribution Non Commercial (CC BY-NC 4.0) license, which permits others to distribute, remix, adapt, build upon this work non-commercially, and license their derivative works on different terms, provided the original work is properly cited, appropriate credit is given, any changes made indicated, and the use is non-commercial. See: http://creativecommons.org/licenses/by-nc/4.0/.

ORCID iD

Xueni Ma http://orcid.org/0000-0001-9635-8370

\section{REFERENCES}

1 Rubin DT, Ananthakrishnan AN, Siegel CA, et al. Acg clinical guideline: ulcerative colitis in adults. Am J Gastroenterol 2019;114:384-413.

2 Guo X, Yan M. Pregnane X receptor polymorphisms and risk of inflammatory bowel disease: a meta-analysis. Immunol Invest 2017;46:566-76.

3 Kaplan GG, Ng SC, . Globalisation of inflammatory bowel disease: perspectives from the evolution of inflammatory bowel disease in the UK and China. Lancet Gastroenterol Hepatol 2016;1:307-16.

4 Peery AF, Dellon ES, Lund J, et al. Burden of gastrointestinal disease in the United States: 2012 update. Gastroenterology 2012;143:1179-87.

5 Alrubaiy L, Rikaby I, Dodds P, et al. Systematic review of healthrelated quality of life measures for inflammatory bowel disease. $J$ Crohns Colitis 2015;9:284-92.

6 Calvert M, Blazeby J, Altman DG, et al. Reporting of patient-reported outcomes in randomized trials: the CONSORT pro extension. JAMA 2013:309:814-22.

7 Bansal D, Bhagat A, Schifano F, et al. Role of patient-reported outcomes and other efficacy endpoints in the drug approval process in Europe (2008-2012). J Epidemiol Glob Health 2015;5:385-95.

8 Ungaro R, Mehandru S, Allen PB, et al. Ulcerative colitis. Lancet 2017;389:1756-70.

9 Sandborn WJ, van Assche G, Reinisch W, et al. Adalimumab induces and maintains clinical remission in patients with moderate-to-severe ulcerative colitis. Gastroenterology 2012;142:e653:257-65.

10 Sandborn WJ, Feagan BG, Marano C, et al. Subcutaneous golimumab induces clinical response and remission in patients with moderate-to-severe ulcerative colitis. Gastroenterology 2014;146:85-95.

11 Kobayashi T, Suzuki Y, Motoya S, et al. First Trough level of infliximab at week 2 predicts future outcomes of induction therapy in ulcerative colitis-results from a multicenter prospective randomized controlled trial and its post hoc analysis. J Gastroenterol 2016;51:241-51.
12 Sandborn WJ, Su C, Sands BE, et al. Tofacitinib as induction and maintenance therapy for ulcerative colitis. N Engl J Med 2017;376:1723-36.

13 Sandborn WJ, Baert F, Danese S, et al. Efficacy and safety of Vedolizumab subcutaneous formulation in a randomized trial of patients with ulcerative colitis. Gastroenterology 2020;158:e12:562-72.

14 Zhou H-Y, Guo B, Lufumpa E, et al. Comparative of the effectiveness and safety of biological agents, tofacitinib, and fecal microbiota transplantation in ulcerative colitis: systematic review and network meta-analysis. Immunol Invest 2021;50:1-15.

15 Singh S, Murad MH, Fumery M, et al. First- and second-line pharmacotherapies for patients with moderate to severely active ulcerative colitis: an updated network meta-analysis. Clin Gastroenterol Hepatol 2020;18:2179-91.

16 Williet N, Sandborn WJ, Peyrin-Biroulet L. Patient-Reported outcomes as primary end points in clinical trials of inflammatory bowel disease. Clin Gastroenterol Hepatol 2014;12:1246-56.

17 Paschos P, Katsoula A, Salanti G, et al. Systematic review with network meta-analysis: the impact of medical interventions for moderate-to-severe ulcerative colitis on health-related quality of life. Aliment Pharmacol Ther 2018;48:1174-85.

18 Leonardi CL, Kimball AB, Papp KA, et al. Efficacy and safety of ustekinumab, a human interleukin-12/23 monoclonal antibody, in patients with psoriasis: 76-week results from a randomised, doubleblind, placebo-controlled trial (Phoenix 1). Lancet 2008;371:1665-74.

19 Feagan BG, Sandborn WJ, Gasink C, et al. Ustekinumab as induction and maintenance therapy for Crohn's disease. N Engl J Med 2016;375:1946-60.

20 Sands BE, Sandborn WJ, Panaccione R, et al. Ustekinumab as induction and maintenance therapy for ulcerative colitis. $N$ Engl J Med 2019;381:1201-14.

21 Sands BE, Peyrin-Biroulet L, Loftus EV, et al. Vedolizumab versus adalimumab for moderate-to-severe ulcerative colitis. N Engl J Med 2019;381:1215-26.

22 Moher D, Shamseer L, Clarke M, et al. Preferred reporting items for systematic review and meta-analysis protocols (PRISMA-P) 2015 statement. Syst Rev 2015;4:1.

23 Hutton B, Salanti G, Caldwell DM, et al. The PRISMA extension statement for reporting of systematic reviews incorporating network meta-analyses of health care interventions: checklist and explanations. Ann Intern Med 2015;162:777-84.

24 Higgins JPT, Altman DG, Gøtzsche PC, et al. The Cochrane collaboration's tool for assessing risk of bias in randomised trials. BMJ 2011;343:d5928.

25 Sterne JAC, Savović J, Page MJ, et al. Rob 2: a revised tool for assessing risk of bias in randomised trials. BMJ 2019;366:14898.

26 DerSimonian R, Laird N. Meta-Analysis in clinical trials revisited. Contemp Clin Trials 2015;45:139-45.

27 Efthimiou O, Rücker G, Schwarzer G, et al. Network meta-analysis of rare events using the Mantel-Haenszel method. Stat Med 2019;38:2992-3012.

28 Salanti G, Ades AE, loannidis JPA. Graphical methods and numerical summaries for presenting results from multiple-treatment metaanalysis: an overview and tutorial. J Clin Epidemiol 2011;64:163-71.

29 Sterne JAC, Sutton AJ, loannidis JPA, et al. Recommendations for examining and interpreting funnel plot asymmetry in meta-analyses of randomised controlled trials. BMJ 2011;343:d4002.

30 Atkins D, Best D, Briss PA, et al. Grading quality of evidence and strength of recommendations. BMJ 2004;328:1490.

31 Zeng Xiantao LW, Sheng L, et al. How to understand and use the grade system correctly. Chinese Journal of Evidence-based Medicine 2011;11:985-90. 